\title{
Simulation of cascading failures in the Eletrosul high-voltage power- grid
}

\author{
T. Carlotto, J.M.V. Grzybowski \\ Universidade Federal da Fronteira Sul - UFFS \\ Av. Dom João Hoffmann, 313, CEP 99.700-000, Erechim, RS. \\ E-mail: jose.grzybowski@uffs.edu.br
}

\begin{abstract}
: a model for cascading failures in power-grid networks is used to study the robustness characteristics of a model of Eletrosul high-voltage power-grid against cascading failures. Based on measures of the electrical centrality and degree, the removal of key nodes is performed in order to explore the dynamical behavior of cascading failures as overloaded generators, substations and transmission lines are tripped offline the grid. The results indicate that major cascading failures follow from the failure of nodes with highest loads and that the existence of operational reserves might not be enough to prevent blackouts when failures occur in key points of the network.
\end{abstract}

\section{Introduction}

The proper functioning of power-grids depends on the continuity of the flow of electricity among generators, substations and consumer centers (nodes) which are wired together by means of transmission lines (links). In this process, the load in each point of the network is subject to dynamical changes, as the energy output from generators must measure up to the demand from consumer centers at any given time. As long as this condition is met, the consumers are supplied and the grid works properly. But, what if a node or link fails? The dynamical and topological features of the power grid network will determine, to a large extent, how the power grid will behave in the face of failures. The more the dynamical and topological characteristics of the network favor stability, efficiency and robustness, the more robust the power-grid will be against malfunctioning. In this context, the complexity and impenetrability of real power grids have inspired the development of simplified models that can retain relevant aspects of the dynamical behavior of electrical networks and allow substantial comprehension of the efficiency drop to which a power-grid is subject when a node fails. A model of cascading failure based on the redistribution of loads after failures was proposed in [1] and used to study the behavior of the Italian power grid [2]. Further studies considered error and attack tolerance in complex networks [3][4] as a means to assess their robustness against random failures and targeted attacks, having concluded the likely effects of such events upon the structural characteristics of scale-free, random networks and real-world networks [4]; as it turns out, most networks seem to be more fragile to failures in the nodes with highest centrality.

In this paper, we apply the model for cascading failures based on the methodology proposed [1] to simulate cascading failures in the graph of Eletrosul transmission system [5]. Based on the results, we confirm the results from previous studies regarding the fragility of the graph against failures of the nodes with highest centrality.

\section{Materials and methods}

The model for cascading failures, as proposed in [1] considers the power-grid as a network with $N$ nodes and $m$ links in which the evolution of the efficiency of transmission over time is based on the flow redistribution after the breakdown of a node. It is assumed that the flow between any two nodes always takes the most efficient path connecting them which, in the electrical context, we take to be that with highest conductance, as inverse to resistance. We consider the resistance of a path to be proportional to its physical length and disregard any reactance of the lines. The modeling give rise to an undirected adjacency matrix from which the load in each node (i.e., its betweenness centrality) is calculated. The efficiency matrix is 
composed by entries in the interval $[0,1]$ and, initially, the efficiency of all existing links is set as $e_{i j}=1$ as all the transmission lines are assumed to be working perfectly. According to [1], the efficiency of a path can modeled as the harmonic composition of the link it goes along, such that the average efficiency of the network is given by

$$
E(G)=\frac{1}{N(N-1)} \sum_{\substack{j=1 \\ j \neq i}}^{N} \frac{1}{d_{i j}}=\frac{1}{N(N-1)} \sum_{\substack{j=1 \\ j \neq i}}^{N} \varepsilon_{i j}
$$

where $d_{i j}$ is the shortest distance between nodes $i$ and $j$ and $\varepsilon_{i j}=1 / d_{i j}$ is the value of the most efficient path [1]. The load upon node $i$ at time $t$, denoted $L_{i}(t)$, is defined as the total number of most efficient paths passing through $i$ at time $t$, where $t$ is a discrete variable. Further, each node is characterized by a capacity that defines the maximum load that it can handle, given by $C_{i}=\eta L_{i}(0)$, where $\eta \geq 1$ is the tolerance parameter of the network. The tolerance parameter is related to the capability of the node to handle overloads, and $L_{i}(0)$ is the initial load of node $i$. The removal of a node simulates a failing electrical substation and it changes the most efficient paths within the network. As such, the efficiency matrix is changed at every iteration due to the redistribution of loads over the network. This effect can be modeled by the iterative rule

$$
e_{i j}(t)=\left\{\begin{array}{l}
e_{i j}(0) \frac{C_{i}}{L_{i}(t)}, \text { if } L_{i}(t)>C_{i} \\
e_{i j}(0), \quad \text { if } L_{i}(t) \leq C_{i}
\end{array}\right.
$$

such that a congestion in node $i$ at time $t$ causes the efficiency of all links passing through it to be reduced, such that energy flow will take alternative paths [1].

Differently from [1], we assume that overloaded links and nodes are shut down, which seems more likely to be the case in electrical networks. After a failure, energy flow is reallocated and handled by remaining links and nodes, iteratively. The dynamics of cascading failures indicate the route of a black-out process initiated due to the failure of a specific node will follow and indicates potential fragilities within the network structure. As the degradation reaches a steady-state, the links and nodes that remain functional correspond to the power-grid in the period post-failure.

The graph of Eletrosul high-voltage power-grid, our object of study, is composed of $N=141$ nodes and $m=180$ links. Its map and topological structure are presented in Figure 1.

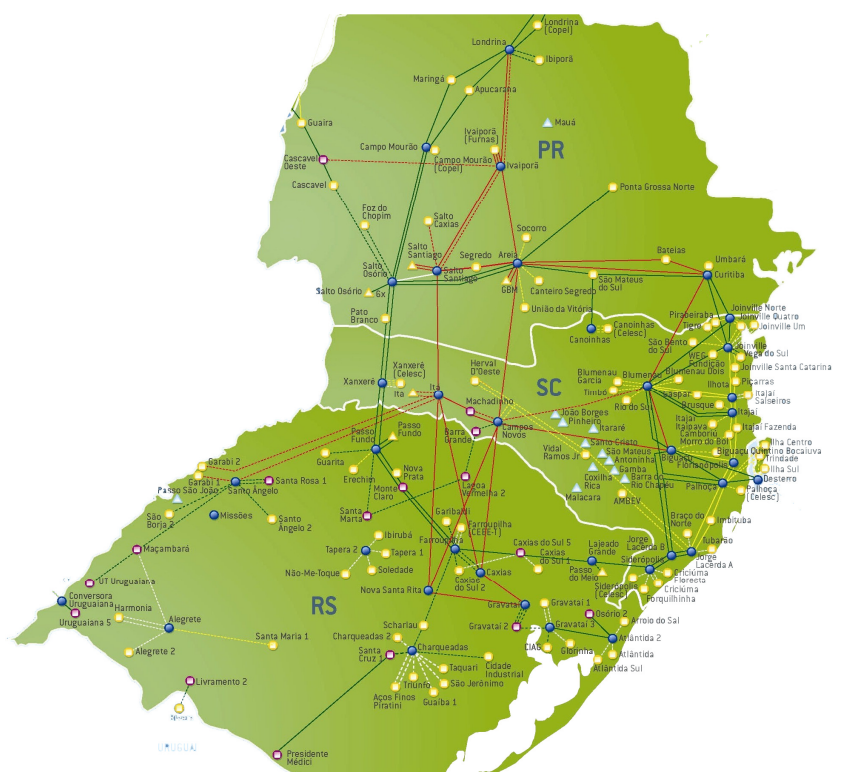

Figure 1 - Eletrosul high-voltage grid, which comprehends three southern Brazilian states. Extracted from [5] and edited by the authors. 
For the numerical simulations, we consider $\eta=1.5$, which means that the network is operating at $2 / 3$ of its capacity in the moment that the failure occurs. The simulations comprises three scenarios: 1) the removal of the node with highest load;2) the removal of the node with highest degree and 3) the removal of a node chosen at random among those whose characteristics are representative of most of the existing nodes, i.e., low degree and load. The detailed characteristics of the nodes removed in each simulation are given in Table 1.

\begin{tabular}{|l|r|r|}
\hline \multicolumn{1}{|c|}{ Node } & \multicolumn{1}{c|}{ Load } & \multicolumn{2}{c|}{ Degree } \\
\hline Highest load & $8,496.4$ & 9 \\
\hline Highest degree & $4,784.5$ & 13 \\
\hline Random & 0.0 & 2 \\
\hline
\end{tabular}

Table 1 - Load and degree characteristics of the nodes subjected to removal.

\section{Results and discussion}

The removal according to scenario 1 (highest load) causes more extensive link breakdowns (the links in red in Figure 2). In such case, the original connected power-grid is broken into three disconnected sub-networks and 38,33\% of the links are disrupted as the cascading process stops. For comparison, the post-failure network induced by the removal according to scenario 2 (highest degree) has $32,22 \%$ of its links degraded. This indicates that the load, which is defined as electrical centrality, is a major indicator of the relative importance of each node, which confirms the results obtained in [4].

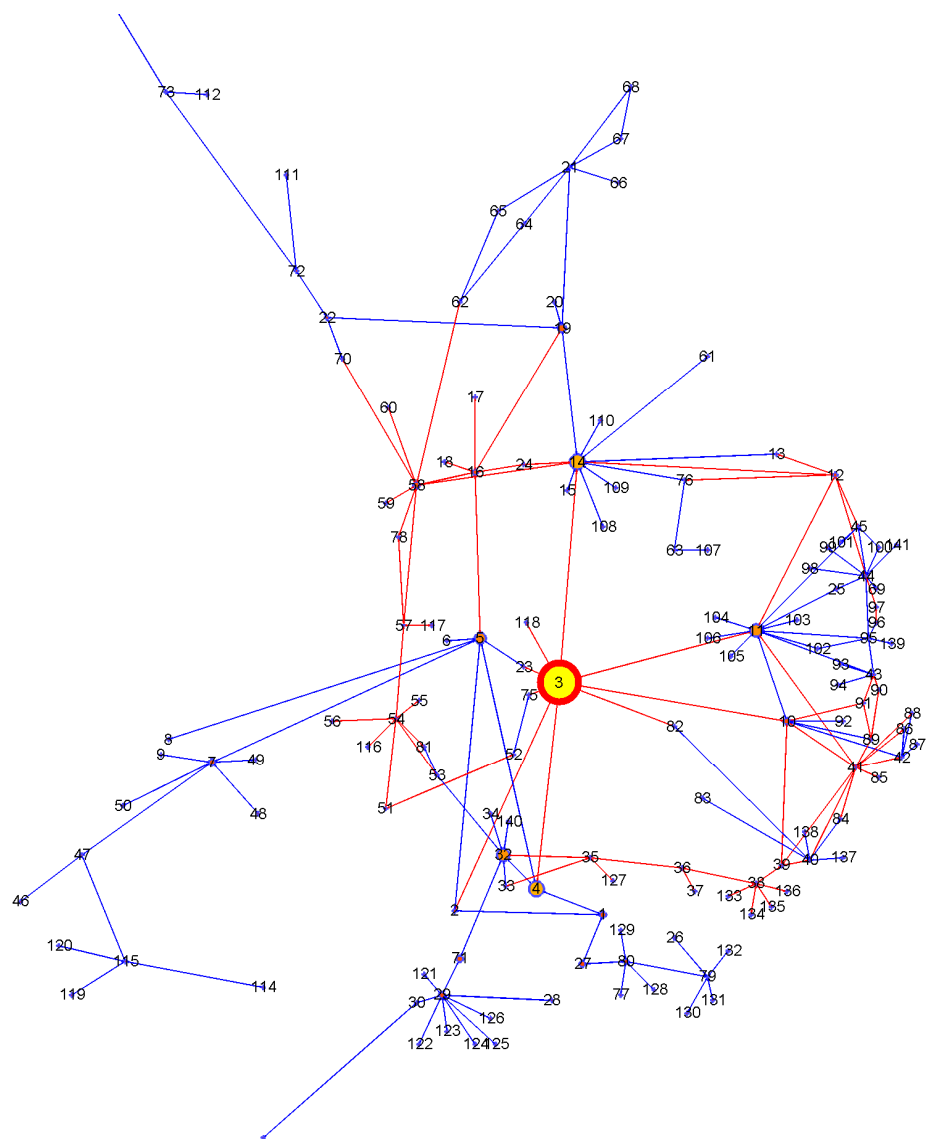

Figure 2 - Cascading failure due to the removal of the node with largest load (circled in red): operational links (in blue) and broken links (in red). 


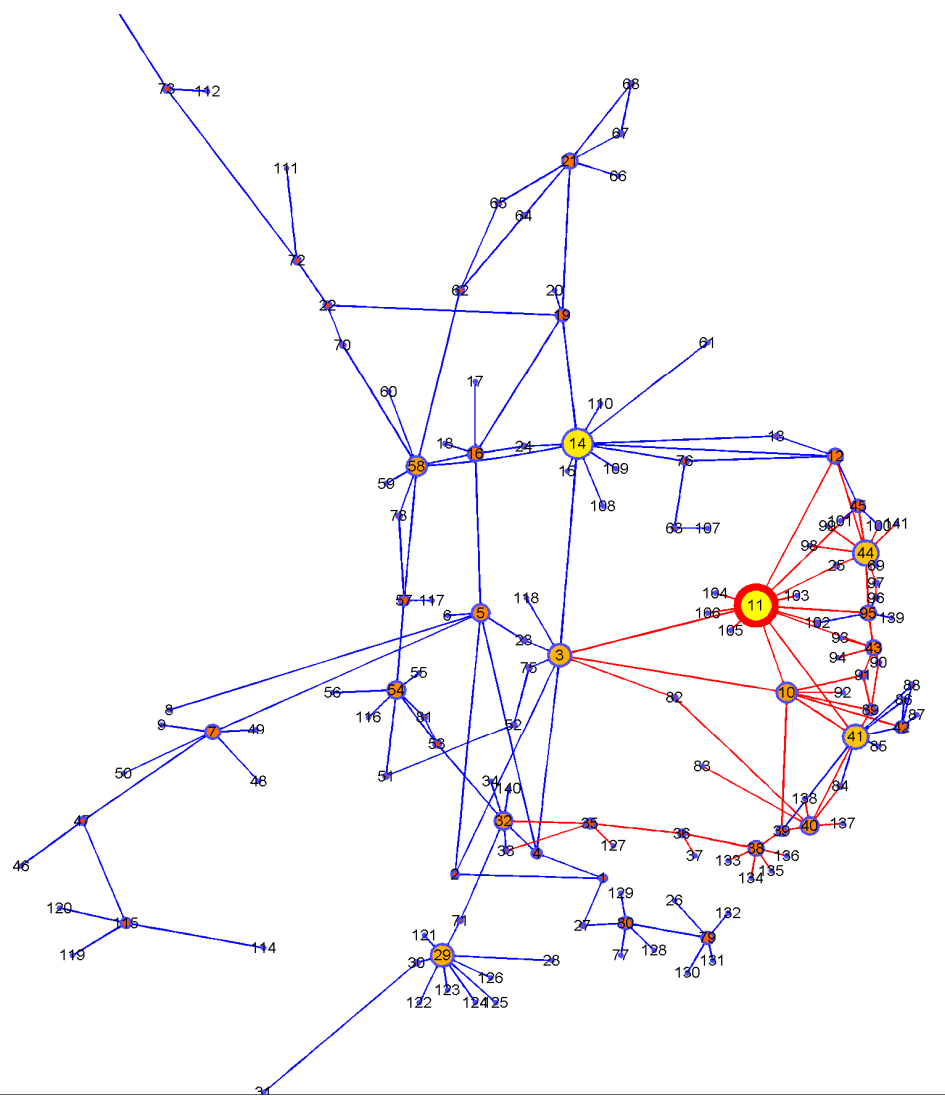

Figure 3 - Cascading failure due to the removal of the node with largest degree (circled in red): operational links (in blue) and broken links (in red).

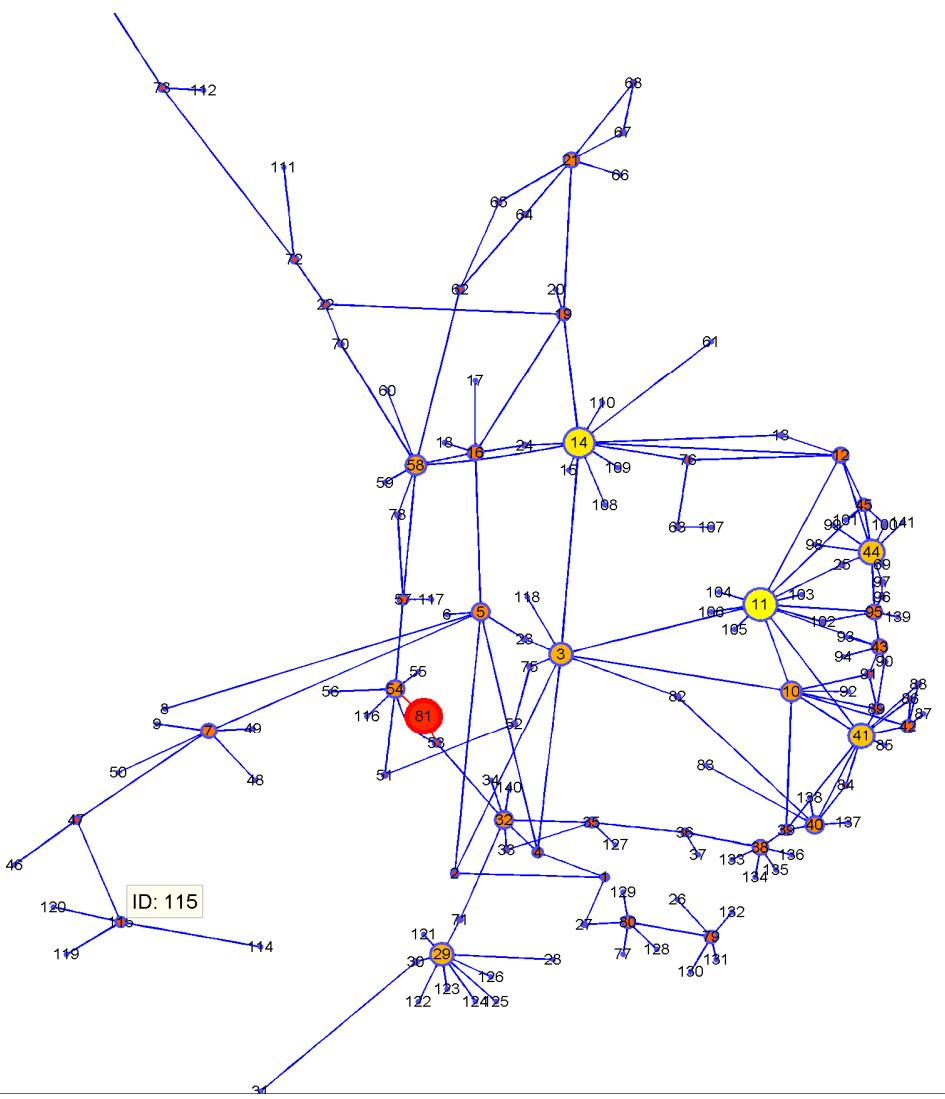

Figure 4 - Cascading failure due to the removal of randomly chosen node (filled in red): operational links (in blue) and broken links (in red). As a result, only the two transmission lines connecting the node to the grid are shut down. 
The post-failure network for scenario 2 is shown in Figure 3 with disabled links in red. Finally, the removal according to scenario 3 resulted in minor damage to the network structure, being the post-failure network disrupted in $1,11 \%$ of its links. The random node happened to have zero load as it is an end-of-line node. Such node happened to be representative of about $60 \%$ of the network nodes, which cause efficiency drops of the same order as they get removed. This shows that nodes with low degree and load are not likely to cause major impacts as they induce only local effects.

Considering a Barabasi-Albert network with $N=2000, K=10000$ and degree distribution $P \sim k^{-\gamma}$ for $\gamma=3$ and the tolerance parameter $\eta=1.5$, the authors in [1] found that efficiency remained completely unaffected by the random removal of a node. We found that this is not true in the case of the network obtained from the topology of Eletrosul high-voltage power-grid. Rather, the removal of any nodes invariably cause overall network efficiency to drop. In fact, efficiency is lost despite the values of the tolerance parameter are set to high values, indicating that the tolerance parameter itself cannot completely compensate the loss of a node.

The simulation of diverse other scenarios showed that the topology of the Eletrosul power-grid is sensitive to the removal of key nodes while random removals cause local effects. It appears to be especially fragile under the removal of nodes with highest betweeness centrality. Further, the tolerance parameter $\eta$, which is related to the existence and availability of operational reserves, was shown to have considerable effects upon the size and scope of cascading failures. However, it does not completely prevent efficiency drops due to the removal of nodes. An interpretation of the results in this context leads to the conclusion that operational reserves can manage failures partially and more connections (transmission lines) might be needed in key points of the network. A question that emerges from this is where to put new nodes such that the efficiency drops are managed to remain at their lowest values.

While theoretical studies of power-grids can provide valuable insights into their dynamical functioning, it should be remarked that the model is a simplification that does not take into account control equipment designed to prevent cascading failures, which are available in real networks and usually handle contingency situations fairly well. On the other hand, simplified models can provide insights into general topological properties influencing the dynamical behavior of power grids which would remain veiled as exceedingly complex models are studied. Further research shall be dedicated to further comprehension of the implications of such topological properties upon the robustness and synchronizability of power grids.

The authors thank FAPERGS for the financial support, grant number 12/1644-2.

Keywords: power-grids, cascading failures, networks, Eletrosul transmission system.

\section{References}

[1] P. Cruciti, V. Latora, M. Marchiori, "Model for cascading failures in complex networks", Phis. Rev. E, vol. 69, 045104 (2004).

[2] P. Crucitti, V. Latora, M. Marchiori. "A topological analysis of the Italian electric power grid”, Physica A 338, p.p. 92-97 (2004).

[3] P. Cruciti, V. Latora, M. Marchiori, A. Rapisarda, "Error and attack tolerance of complex networks", Physica A, vol. 340, p.p. 388-394 (2004).

[4] M. Piraveenan, S. Uddin, K. Shing, K. Chung. "Measuring topological robustness of networks under sustained targeted attacks". Proceedings of the 2012 IEEE/ACM International Conference on Advances in Social Networks Analysis and Mining, 2012.

[5] Map of the Electrical Transmission System, Eletrosul - Centrais Elétricas. Available online at http://www.eletrosul.gov.br/arquivos/Sistema Transmissao 04SET2009.jpg, (2009). 